\title{
Effects of vacuum sealing drainage to improve the therapeutic effect in patients with orthopedic trauma and to reduce post-operative infection and lower-limb deep venous thrombosis
}

\author{
LEI ZHANG ${ }^{1,2 *}$, YAO ZHAO ${ }^{3 *}$, YAN LU $^{2}$, PINGPING HE ${ }^{4}$, PENG ZHANG ${ }^{1}$, ZHANHUI LV $^{2}$ and YIXIN SHEN ${ }^{1}$ \\ ${ }^{1}$ Department of Orthopaedics, The Second Affiliated Hospital of Soochow University, Suzhou, Jiangsu 215006; \\ ${ }^{2}$ Department of Orthopaedics, Binzhou Central Hospital Affiliated to Binzhou Medical University, Binzhou, Shandong 251700; \\ ${ }^{3}$ Department of Orthopedics, Shandong Provincial ENT Hospital Affiliated to Shandong University, \\ Jinan, Shandong 250000; ${ }^{4}$ Department of Clinical Pharmacy, Binzhou Central Hospital \\ Affiliated to Binzhou Medical University, Binzhou, Shandong 251700, P.R. China
}

Received July 1, 2019; Accepted February 10, 2020

DOI: $10.3892 /$ etm.2020.8941

\begin{abstract}
The present study investigated the effects of vacuum sealing drainage (VSD) to improve the therapeutic efficacy in patients with orthopedic trauma (OT) and reduce post-operative infection and lower-limb deep venous thrombosis (DVT) through a retrospective analysis. A total of 76 patients with OT treated at our hospital were selected for observation. The patients were divided into the control group $(\mathrm{CG} ; \mathrm{n}=37)$ and the experimental group (EG; $n=39$ ) according to the treatment administered. For patients in the $\mathrm{CG}$, routine dressing changes were applied. Patients in the EG underwent VSD treatment. The dressing change frequency, time between the first and second operation, hospital stay, treatment efficacy, wound healing time, interleukin-6 (IL-6) serum level, tumor necrosis factor- $\alpha$ (TNF- $\alpha$ ) serum level, incidence of post-operative infection and incidence of lower-limb DVT were compared between the two groups. The dressing change frequency in the EG was less than that in the CG. The time between the first and second operation and hospital stay were shorter in the EG than in the $\mathrm{CG}(\mathrm{P}<0.05)$. The total effective rate in the EG was $97.44 \%$, which was higher than that in the CG $(78.38 \%$; $\mathrm{P}<0.05)$. The wound healing time in the EG was $1.72 \pm 0.73$ weeks and shorter than that in the CG $(2.23 \pm 0.85$ weeks; $\mathrm{P}<0.05)$. With the progression of treatment, the serum IL-6 and TNF- $\alpha$ levels decreased in the two groups, but the levels
\end{abstract}

Correspondence to: Dr Yixin Shen, Department of Orthopaedics, The Second Affiliated Hospital of Soochow University, 1055 Sanxiang Road, Suzhou, Jiangsu 215006, P.R. China

E-mail: yyyishee@163.com

*Contributed equally

Key words: vacuum sealing drainage, orthopedic trauma, postoperative infection, lower-limb deep venous thrombosis in the EG were lower than those in the $\mathrm{CG}(\mathrm{P}<0.05)$. The incidence of post-operative infection and lower-limb venous thrombosis in the EG were 7.69 and $0.00 \%$, respectively, and lower than those in the CG (27.03 and $13.01 \%$, respectively; $\mathrm{P}<0.05)$. In the treatment of OT, VSD may reduce the dressing change frequency, shorten the operation time and hospital stay, accelerate wound healing and reduce post-operative infection and lower-limb DVT. Thus, the VSD treatment method is worthy of promotion and implementation in clinic.

\section{Introduction}

The routine treatment of orthopedic trauma (OT) frequently involves debridement, washing, dressing changes, antibiotic treatment to control infection and flap surgery after the granulation tissue has grown plump. For certain cases of OT with large wounds and seriously injured soft tissues, however, the dressing change is required more frequently if the conventional treatment is used. In addition, the wound surface exposure time is long, the necrotic tissue and toxins are absorbed and the healing time is prolonged. Furthermore, the risk of infection increases $(1,2)$. Trauma, a period of inactivity and vascular wall injury are risk factors for lower-limb deep venous thrombosis (DVT) in patients with OT (3). Patients with long-term wound disunion require a long period of inactivity due to the prolonged course of the condition. Thus, lower-limb DVT is more likely to occur (4). Therefore, accelerating the wound healing process and promoting the recovery of patients with OT may have a significant impact to reduce infection and lower-limb DVT.

Vacuum sealing drainage (VSD) refers to the use of continuous vacuum suction to seal the wound. It enables an easier discharge of the exudate and provides a better clearance effect compared with conventional treatment (5). Application of a VSD technique may completely remove exudate, necrotic fluid and bacteria from the wound surface through a continuous vacuum, removing local dead space, reducing tissue edema 
and making granulation tissue easier to grow. At the same time, vacuum aspiration may deform wound cells, increase the synthesis of proteins and biological macromolecules between cells and accelerate cell proliferation. In addition, excess fluid between tissues may be further extracted, thus reducing tissue edema, promoting local blood circulation and facilitating wound healing. A previous study has indicated that VSD may promote wound healing in OT and improve the survival rate of transplanted skin (6). In the present study, VSD was used for the treatment of patients with OT. The effects to reduce infection and lower-limb DVT were observed and reported below.

\section{Materials and methods}

General information. The clinical data of 76 patients with OT admitted to The Second Affiliated Hospital of Soochow University (Suzhou, China) from January 2016 to January 2019 were retrospectively analyzed and categorized into a control group $(\mathrm{CG} ; \mathrm{n}=37)$ and an experimental group $(\mathrm{EG} ; \mathrm{n}=39)$ according to the treatment administered. The general patient information exhibited no differences between the two groups and the groups were comparable $(\mathrm{P}>0.05$; Table I). The present study was approved by the ethics committee of The Second Affiliated Hospital of Soochow University (Suzhou, China). The inclusion criteria were as follows: i) Age, 18-70 years; ii) lumbosacral or lower-limb trauma; iii) primary suture not practical or possible, and iv) signed informed consent form. The exclusion criteria were as follows: i) Serious infection secondary to fresh trauma, ii) infectious diseases as complications and iii) serious complications.

Methods. The patients in each group underwent debridement and repair of damaged blood vessels, nerves and tendons. For patients with fracture, fracture reduction and fixation were performed. All patients were treated with antibiotics to control infection. Routine dressing changes once every 1-2 days were applied to patients in the CG. If the wound was large and deep, a drainage strip was placed. After the granulation tissue had grown plump, wound suture, skin grafting or flap transfer surgery was performed according to the wound condition. Patients in the EG underwent VSD treatment (7). After routine debridement, according to the size and shape of the wound, a vacuum therapeutic sponge adsorption pad and isolation pad of a suitable size was made with sterile scissors (Jiangsu Suzhong Pharmaceutical Group, Co., Ltd.) to cover the wound surface to ensure complete coverage, and the isolation pad was placed between the adsorption pad and the wound surface. Depending on the amount of exudate on the wound surface and the depth of the wound, the adsorption sponge was able to be completely inserted into the wound surface, with the outer surface covered with the adsorption pad. The wound surface was closed with a medical biological semipermeable membrane (Xiamen Shanxing Hanfang Biotechnology Co., Ltd.) tightly attached to the skin, with the outer edge of the membrane covering the normal skin outside of the wound edge by at least $>3 \mathrm{~cm}$. Medical tape was used to seal the wound again to ensure that the wound was closed. One end of the silicone tube (Dongguan Ruixiang Precision Silicone Products Co., Ltd.) was connected to the dressing and the other end was connected to the vacuum suction device (Suzhou Oris Medical Supplies Co., Ltd.) to ensure a good seal and no air leakage. The vacuum pressure was set between -450 and $-125 \mathrm{mmHg}$. At the same time, an antibacterial agent was selected after a pathogenic test for anti-infection treatment. If the wound was deep and large, the dressing was replaced 2-3 times depending on the patient's situation. The dressing maintenance time was up to 10 days. Wound suture and skin grafting were performed when the granulation tissue was plump. If the granulation tissue was not plump and depending on the patient's situation, a skin flap transfer was performed.

Outcome measures. i) The dressing change frequency (the numbers of dressing change during wound healing), dressing maintenance time (duration of dressing in the wound after dressing change), 2nd operation time (the time between the first operation and the second operation) and duration of hospital stay (complete hospital stay) were recorded; ii) with respect to efficacy, 'cure' was defined as the healing of all wounds within two weeks with good epidermal coverage. 'Improvement' was defined as a significantly reduced wound area, the survival of most wound skin tissues, decreased secretions and good growth of fresh and healthy granulation tissue, although the dressing was still required to be replaced. In cases of improvement, the wound healed after skin grafting. The treatment was deemed ineffective when the wound area did not decrease or expand, the wound skin was necrotic, the secretions increased and there was no healthy granulation. The total effective rate was calculated as $100 \%$ minus the ineffective rate. Wound healing and evaluation were evaluated by the same attending physician with $>5$ years of clinical experience to reduce errors. iii) Wound healing time was recorded. iv) Venous blood $(3 \mathrm{ml})$ was sampled prior to treatment, at 5 days after VSD treatment and at 10 days after VSD treatment. The levels of serum interleukin-6 (IL-6) and tumor necrosis factor- $\alpha$ (TNF- $\alpha$ ) were determined by ELISA. All kits were purchased from R\&D Systems. The presence of infection and/or lower-limb DVT was observed. The signs of lower-limb DVT included the presence of limb swelling, sharp pain, shank and femoral triangle tenderness, dark-red skin, elevated skin temperature, varicosity and positive results in the straight-leg and ankle-extension tests diagnosed using ultrasound and venography. The manifestations of infection were a red and swollen wound, heat pain and wound sinus after abscess formation diagnosed by bacterial culture and pathological tissue examination.

Statistical analysis. Data were analyzed with SPSS statistical software (version 23.0; IBM Corp.). The measurement data were expressed as the mean \pm standard deviation. Independent-samples t-test was used for the analysis of differences in measurement data. Enumeration data were expressed as $\mathrm{n}(\%)$ and the $\chi^{2}$ test was adopted for the analysis of differences between groups. $\mathrm{P}<0.05$ was considered to indicate a statistically significant difference.

\section{Results}

Comparability of clinical data. In EG, there were 22 males and 17 females, with an average age of $41.65 \pm 7.21$ years; In $\mathrm{CG}$, there were 20 males and 17 females, with an average age 
Table I. Comparison of general information.

\begin{tabular}{|c|c|c|c|c|}
\hline Item & $\begin{array}{l}\text { Experimental group } \\
\qquad(\mathrm{n}=39)\end{array}$ & $\begin{array}{l}\text { Control group } \\
\qquad(\mathrm{n}=37)\end{array}$ & $\chi^{2} / \mathrm{t}$ value & P-value \\
\hline Sex & & & 0.043 & 0.836 \\
\hline Male & $22(56.41 \%)$ & $20(54.05 \%)$ & & \\
\hline Female & $17(43.59 \%)$ & $17(45.95 \%)$ & & \\
\hline Age (years) & $34-49(41.65 \pm 7.21)$ & $35-48(42.37 \pm 7.39)$ & 0.431 & 0.669 \\
\hline Wound type & & & 0.164 & 0.686 \\
\hline Fresh & $27(69.23 \%)$ & $24(64.86 \%)$ & & \\
\hline Old & $12(30.77 \%)$ & $13(35.14 \%)$ & & \\
\hline Trauma site & & & 0.018 & 0.895 \\
\hline Lower limb & $29(74.36 \%)$ & $28(75.68 \%)$ & & \\
\hline Lumbosacral portion & $10(25.64 \%)$ & $9(24.32 \%)$ & & \\
\hline Cause & & & 0.676 & 0.879 \\
\hline Traffic & $17(43.59 \%)$ & $18(48.65 \%)$ & & \\
\hline Crushing & $13(33.33 \%)$ & $11(29.73 \%)$ & & \\
\hline Falling & $7(17.95 \%)$ & $5(13.51 \%)$ & & \\
\hline Others & $2(5.13 \%)$ & $3(8.11 \%)$ & & \\
\hline
\end{tabular}

Values are expressed as $\mathrm{n}(\%)$ or the mean \pm standard deviation.

Table II. Comparison of efficacy.

\begin{tabular}{lcccc}
\hline & $\begin{array}{c}\text { Experimental } \\
\text { group } \\
(\mathrm{n}=39)\end{array}$ & $\begin{array}{c}\text { Control } \\
\text { group } \\
(\mathrm{n}=37)\end{array}$ & t value & P-value \\
\hline Efficacy & $26(66.67)$ & $19(51.35)$ & & \\
Cure & $12(30.77)$ & $10(27.03)$ & & \\
Improvement & $1(2.56)$ & $8(21.62)$ & & \\
Ineffectiveness & $38(97.44)$ & $29(78.38)$ & 4.906 & 0.01 \\
Total effective rate & & & & \\
\hline Values are expressed as n (\%). & & & & \\
\hline
\end{tabular}

of $42.37 \pm 7.39$ years. There were no significant differences detected in sex, average age, wound type, trauma region or cause of trauma between the two groups $(\mathrm{P}>0.05)$. The clinical data were comparable between the two groups (Table I).

Acceleration of recovery time. The dressing change frequency in the EG was less than that in the CG $(\mathrm{P}<0.05)$. The 2nd operation time and hospital stay were shorter in the EG than in the $\mathrm{CG}(\mathrm{P}<0.05)$. The dressing maintenance time was longer in the EG than in the CG $(\mathrm{P}<0.05)$. These results indicate that VSD markedly reduced the dressing change frequency, increased the dressing maintenance time and shortened the 2nd operation time and hospital stay. Thus, the recovery of patients with OT was accelerated (Fig. 1).

Improvement of therapeutic effect. The total effective rate in the EG was higher than that in the CG $(\mathrm{P}<0.05$; Table II). This implies that VSD is able to promote wound healing and improve the clinical efficacy of treatment.
Table III. Comparison of wound healing time.

\begin{tabular}{|c|c|c|c|c|}
\hline Item & $\begin{array}{l}\text { Experimental } \\
\text { group } \\
(n=39)\end{array}$ & $\begin{array}{l}\text { Control } \\
\text { group } \\
(n=37)\end{array}$ & $\chi^{2 / t}$ value & $\mathrm{P}$-value \\
\hline $\begin{array}{l}\text { Wound healing } \\
\text { duration (weeks) }\end{array}$ & & & 8.902 & 0.031 \\
\hline 1 & $18(46.15)$ & $9(24.32)$ & & \\
\hline 2 & $15(38.46)$ & $11(29.73)$ & & \\
\hline 3 & $5(12.82)$ & $15(40.54)$ & & \\
\hline$>3$ & $1(2.56)$ & $2(5.41)$ & & \\
\hline $\begin{array}{l}\text { Average healing } \\
\text { time (weeks) }\end{array}$ & $1.72 \pm 0.73$ & $2.23 \pm 0.85$ & 2.81 & 0.006 \\
\hline
\end{tabular}

Values are expressed as $\mathrm{n}(\%)$ or the mean \pm standard deviation.

Shortening of wound healing time. The wound healing time in the EG was shorter than that in the CG $(\mathrm{P}<0.05$; Table III). This suggests that VSD is able to shorten the wound healing time and accelerate the recovery of patients with OT.

Reduction of the serum levels of inflammatory factors. With the progression of the treatment, the levels of serum IL- 6 and TNF- $\alpha$ in the two groups decreased at days 5 and 10 after treatment, respectively, but the levels in the EG were lower than those in the $\mathrm{CG}(\mathrm{P}<0.05)$. This result indicates that VSD is able to decrease the serum levels of inflammatory factors, reduce wound inflammation and promote wound healing (Fig. 2).

Reduction of the incidence of post-operative infection and lower-limb venous thrombosis. There were 5 cases of 

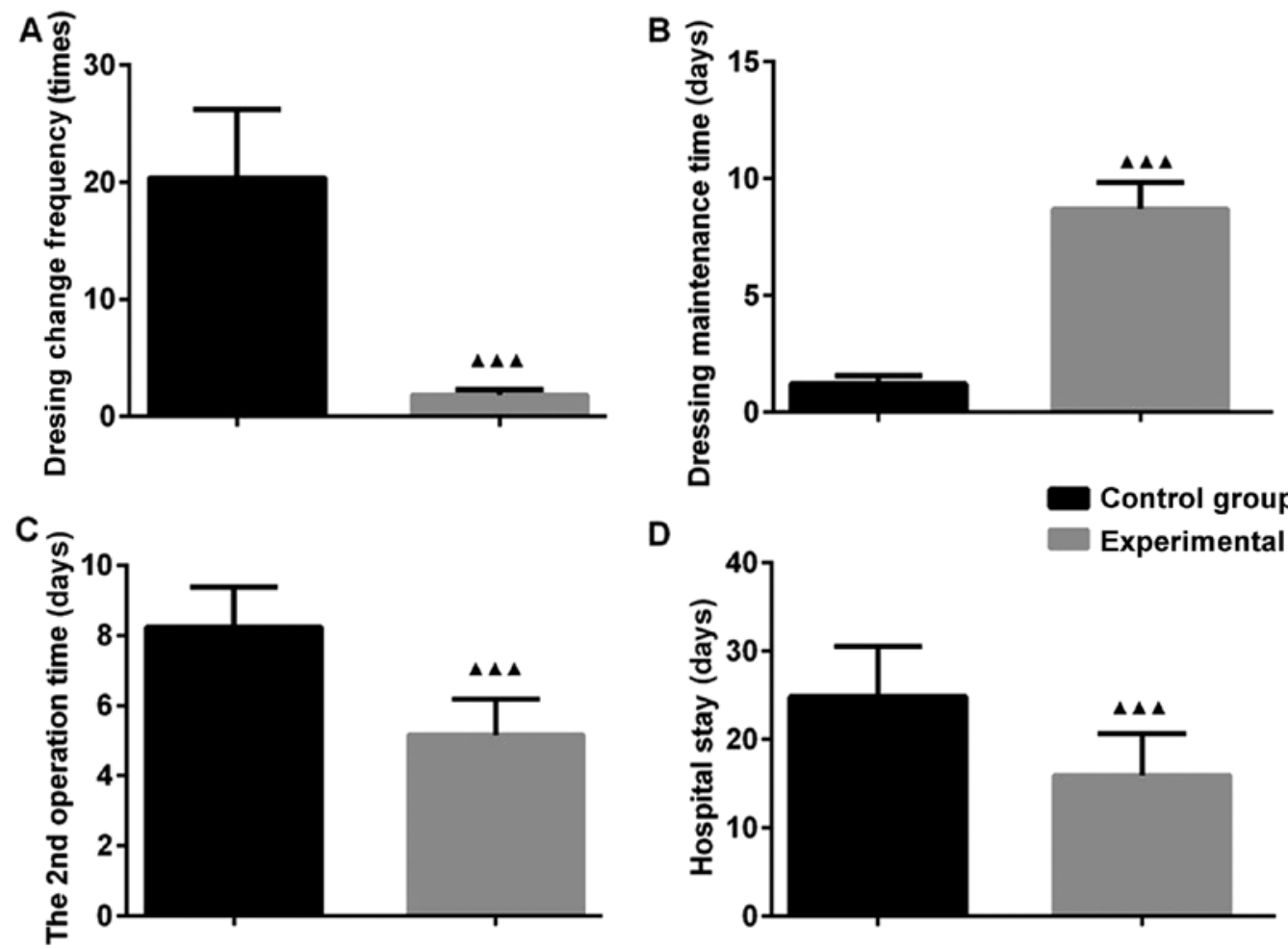

D

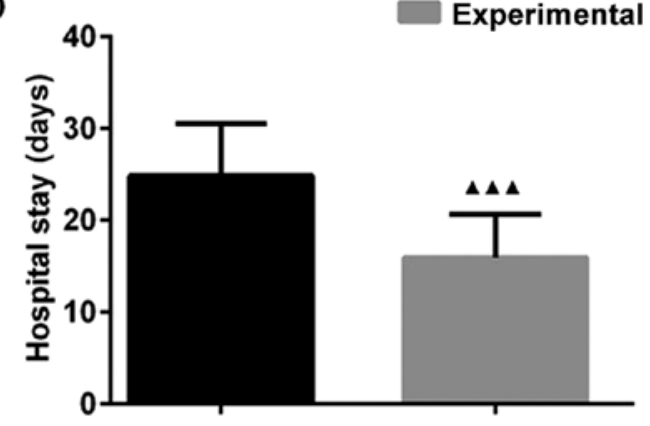

Figure 1. Vacuum sealing drainage is able to accelerate the recovery time of patients with orthopedic trauma. (A) Dressing change frequency; (B) dressing maintenance time; (C) 2nd operation time; (D) hospital stay. ${ }^{\wedge} \mathrm{P}<0.001$ compared with the control group.
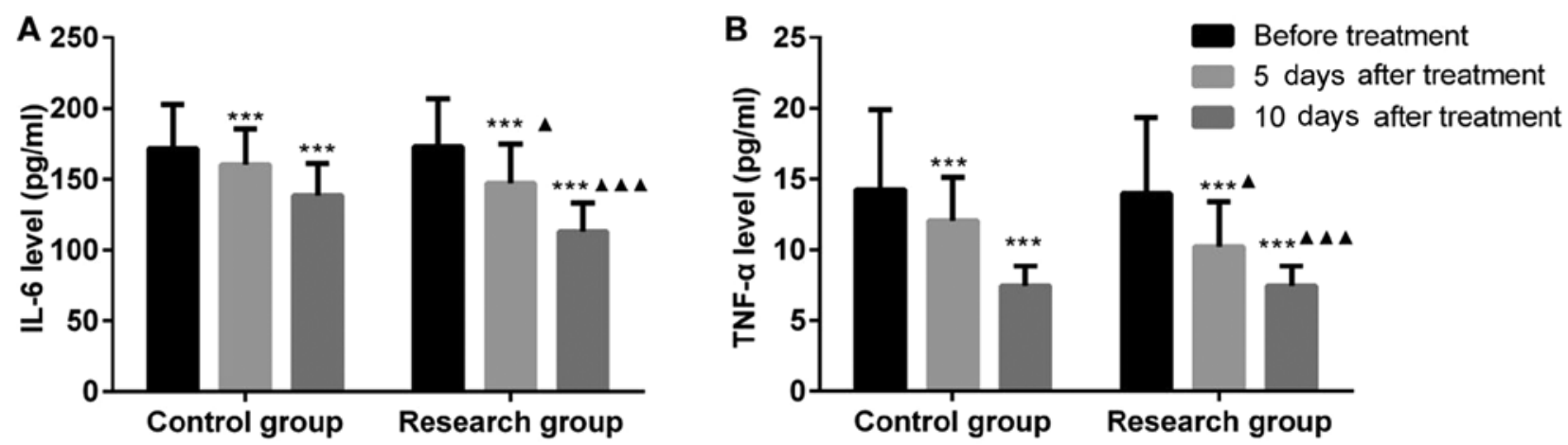

Figure 2. Vacuum sealing drainage is able to reduce the level of inflammatory factors in serum. (A) IL-6 level; (B) TNF- $\alpha$ level. ${ }^{* * *} \mathrm{P}<0.001$ compared with prior to treatment; ${ }^{\wedge} \mathrm{P}<0.05,{ }^{\wedge}{ }^{\wedge} \mathrm{P}<0.001$ compared with the control group at the same time-point. TNF, tumor necrosis factor; IL, interleukin.

lower-limb venous thrombosis in the $\mathrm{CG}$, and no case lower-limb venous thrombosis in the EG was observed. The incidence of post-operative infection and lower-limb venous thrombosis in the EG were lower than those in the $\mathrm{CG}(\mathrm{P}<0.05)$. This implies that VSD may reduce the risk of post-operative infection and lower-limb venous thrombosis (Table IV).

\section{Discussion}

The number of patients with OT has increased with socioeconomic developments. The repair of severely traumatized tissue has become the emphasis and may cause difficulty in the treatment of OT. In certain patients with OT with various types of injury, including skin soft tissue injury, nerve injury and vascular injury, the trauma is difficult to treat due to serious contamination, a deep and large wound and a large amount of exudates. Routine dressing changes may cause considerable pain and a psychological burden in such patients. In addition, the therapeutic effect is frequently unsatisfactory, the dressing requires to be frequently replaced, wound healing is slow and treatment is more difficult once infection occurs (8). A clinical study in 1,196 patients with OT reported an incidence of infection of $2.01 \%$ (9). Open wound, wound drainage, wound contamination and a long hospital stay may increase the risk of wound infection (10). If primary suture cannot be performed in patients with OT, the recovery from trauma may be difficult and a large wound may remain. Furthermore, the connective tissue is exposed during healing without protective epithelial tissue. Delayed nonunion may occur if the wound is not properly treated. This will confer a greater psychological and economic burden to the patients. In the present study, VSD was used for the treatment of OT. The results suggested that VSD 
Table IV. Comparison of the incidence of post-operative infection and lower limb deep venous thrombosis.

\begin{tabular}{lcccr}
\hline Event & $\begin{array}{c}\text { Experimental group } \\
(\mathrm{n}=39)\end{array}$ & $\begin{array}{c}\text { Control group } \\
(\mathrm{n}=37)\end{array}$ & $\chi^{2}$ value & P-value \\
\hline Post-operative infection & $3(7.69)$ & $10(27.03)$ & 5.006 & 0.025 \\
Lower limb venous thrombosis & $0(0)$ & $5(13.51)$ & 3.656 & 0.018 \\
\hline
\end{tabular}

is able to reduce the dressing change frequency, shorten the operation time and hospital stay, accelerate the wound healing process and reduce post-operative infection and lower-limb DVT.

The materials used in VSD are polyethylene/ethanol hydrated seaweed salt foam and polyurethane film, which produce a semipermeable membrane. During treatment, the VSD material is tailored so that its shape and area match those of the wound. Thereafter, a tailored VSD dressing is used to completely cover the wound. A drainage tube is connected and direct contact between the drainage tube and the wound is avoided. After connecting the vacuum suction device, negative pressure is established so that the exudate may be suctioned out. Thereby, the bacteria, toxin and pus on the wound surface may be removed (11). Furthermore, VSD is also able to effectively clear the dead cavity and eliminate the survival environment for pathogens. Thus, a good microenvironment is provided for wound repair, which effectively prevents infection and promotes wound healing (12). The continuous suction of exudate during VSD treatment may obviate frequent dressing changes and reduce the patient's pain (13). In addition, a relatively sealed, clean and dry negative-pressure environment is formed after the wound is covered with the VSD dressing. This contributes to the dilation of the capillaries and accelerates and promotes the growth of granulation tissue (14). Negative pressure helps in controlling local edema, removing inflammatory mediators, reconstructing blood vessels, forming granulation and reducing the biological load of the wound (15). According to relevant studies $(16,17)$, VSD has achieved good results in the treatment of scald wounds and avulsion injuries of limb skin and soft tissues. It may effectively promote wound healing and improve the quality of healing. The results of the present study indicated that the dressing change frequency in the EG was less than that in the CG. The 2nd operation time and hospital stay were shorter in the EG than in the CG. A previous study has also suggested that VSD may effectively shorten the hospital stay and reduce the dressing change frequency compared with traditional pressure dressings in the treatment of degloved skin injury (18). This result is similar to that of the present study and indicates that VSD is able to markedly reduce the dressing change frequency, accelerate wound healing and shorten the 2nd operation time and hospital stay in the treatment of OT without primary suture. The effect was better than that of routine dressing changes. In the present study, the total effective rate in the EG was $97.44 \%$, which was higher than the rate of $78.38 \%$ in the CG. A previous study reported a total effective rate of $98 \%$ in 60 patients with OT and infected wounds treated with VSD (19). This rate was higher than that in the traditional dressings group (75\%). These results are consistent with those of the present study, which indicated that the wound healing time in the VSD group was markedly shorter than that in the routine dressings group. Kaushik et al (20) reported that the complete wound closure time and hospital stay were 12.5 and 17.3 days, respectively, after the contaminated trauma wound was treated with negative-pressure wound therapy. These were markedly shorter than the corresponding durations of 21.4 and 23.8 days in the conventional gauze dressing group, respectively. These results are consistent with those of the present study and imply that VSD is able to accelerate wound healing and shorten the wound healing time.

Trauma may cause an inflammatory response. The release of inflammatory factors into the blood may lead to an abnormal increase in serum inflammatory factors. Upregulation of serum IL- 6 and TNF- $\alpha$ expression is observed in patients with OT. Such upregulation is closely linked to the severity of the trauma (21). The results of the present study indicated that the expression of serum IL- 6 and TNF- $\alpha$ decreased in the two groups with the progression of the treatment, but that the downregulation was more obvious in the EG. This indicates that VSD was able to markedly reduce the degree of inflammatory response and improve the condition of patients with OT. Post-operative infection is a common complication in OT. The post-operative infection rate of patients with open wounds was reported to be up to $25-35 \%$ (22). In the present study, the post-operative infection rate in the EG was $7.69 \%$, which was significantly lower than that in the CG (27.03\%). The reason may be that VSD promotes blood circulation in the wound. In addition, the degree of wound edema is improved; wound bacteria and their products are effectively eliminated; necrotic tissue and abscess are removed; the wound is kept clean; and bacterial proliferation is inhibited. The sealed environment formed by the VSD dressing is able to temporarily isolate the wound from the external environment, thus avoiding wound exposure. Furthermore, the risk of infection is reduced (23). Lower-limb DVT is one of the serious complications in patients with OT. The manifestations are limb swelling, pain, varicosity, exposure and muscle tenderness (24). Patients with OT are usually subjected to violent forces and experience serious traumatic extrusion. After the trauma, a long period of inactivity is required. This leads to poor venous circulation, serious vessel congestion or damaged vascular wall, an activated coagulation mechanism, platelet aggregation and changes in hemodynamics. As a result, lower-limb DVT frequently occurs (25). The present study indicated that the incidence of lower-limb DVT was lower in the EG than in the CG. The reason may be that VSD is able to effectively shorten the wound healing time, reduce the period of inactivity and lower the occurrence and risk of lower-limb DVT; however, the specific mechanisms require to be further investigated. 
There are certain limitations to this study. All patients were treated with antibiotics, but no statistical analyses were performed on the effect of different antibiotics on wound healing. This is required to be further observed in a future study.

In conclusion, VSD in the treatment of OT may reduce the dressing change frequency, shorten the operation time and hospital stay, accelerate wound healing and reduce post-operative infection and lower-limb venous thrombosis. Therefore, the VSD treatment method is worthy of promotion and implementation in clinic.

\section{Acknowledgements}

Not applicable.

\section{Funding}

No funding was received.

\section{Availability of data and material}

All data generated or analyzed during this study are included in this published article.

\section{Authors' contributions}

LZ, YZ and YS conceived the study and designed the experiments. YL and PH contributed to the data collection, PZ and ZL performed the data analysis and interpreted the results. LZ and YZ wrote the manuscript. YS contributed to the critical revision of the article. All authors read and approved the final manuscript.

\section{Ethics approval and consent to participate}

All patients provided written informed consent. The present study was approved by the Ethics Committee of the Second Affiliated Hospital of Soochow University (Suzhou, China).

\section{Patient consent for publication}

Not applicable.

\section{Competing interests}

The authors declare that they have no competing interests.

\section{References}

1. Mannino BJ, Pullen MW and Gaines R: Preventing seal leak during negative pressure wound therapy near external fixators: A Technical tip. J Orthop Trauma 31: e101-e102, 2017.

2. Chagas MQ, Costa AM, Mendes PH and Gomes SC: Analysis of surgical site infections in pediatric patients after orthopedic surgery: A case-control study. Rev Paul Pediatr 35: 18-24, 2017.

3. Walker ME, Tsay C, Broer PN, Zhu VZ, Sturrock T, Ng R, Scoutt LM, Thomson JG and Kwei SL: A prospective, randomized-controlled pilot study comparing closed suction versus negative pressure drains for panniculectomy patients. J Plast Reconstr Aesthet Surg 71: 438-439, 2018.

4. Webb LX: The impact of negative pressure wound therapy on orthopaedic infection. Orthop Clin North Am 48: 167-179, 2017.
5. Davis J, Caruso DM, Foster KN and Matthews MR: A novel approach to sealing the denuded dermis of the abdominal wall with a negative pressure wound device after a decompressive laparotomy. J Burn Care Res 39: 838-842, 2018.

6. Mohsin M, Zargar HR, Wani AH, Zaroo MI, Baba PU, Bashir SA, Rasool A and Bijli AH: Role of customised negative-pressure wound therapy in the integration of split-thickness skin grafts: A randomised control study. Indian J Plast Surg 50: 43-49, 2017.

7. Liu F, Luo Q and Liang Z: The principle of vacuum-assisted closure and wound repair. Chin J Inj Repair Wound Healing 3: 487-492, 2008

8. Engelhardt M, Rashad NA, Willy C, Müller C, Bauer C, Debus S and Beck T: Closed-incision negative pressure therapy to reduce groin wound infections in vascular surgery: A randomised controlled trial. Int Wound J 15: 327-332, 2018.

9. Robert N: Negative pressure wound therapy in orthopaedic surgery. Orthop Traumatol Surg Res 103: S99-S103, 2017.

10. Bcc R and Wenke JC: An effective negative pressure wound therapy-1 compatible local antibiotic delivery device. J Orthop Trauma 31: 1, 2017.

11. Green T, Kavros S, Springer S, Drez D Jr, McCabe M and Gremillion J: Team approach: Complex dermal wound-healing utilizing negative-pressure wound therapy (NPWT) in orthopaedic trauma. JBJS Rev 6: e1, 2018.

12. Nam D, Sershon RA, Levine BR and Della Valle CJ: The use of closed incision negative-pressure wound therapy in orthopaedic surgery. J Am Acad Orthop Surg 26: 295-302, 2018.

13. Chang FS, Chou C, Hu CY and Huang SH: Suture technique to prevent air leakage during negative-pressure wound therapy in fournier gangrene. Plast Reconstr Surg Glob Open 6: e1650, 2018.

14. Shim HS, Choi JS and Kim SW: A role for postoperative negative pressure wound therapy in multitissue hand injuries. BioMed Res Int 2018: 3629643, 2018

15. Bazaliński D, Więch P, Kaczmarska D, Sałacińska I and Kózka M: Use of controlled negative pressure in management of phlegmon caused by fulminant complication of pressure wound: A case report. Medicine (Baltimore) 97: e11319, 2018.

16. Angspatt A, Laopiyasakul T, Pungrasmi P and Suwajo P: The role of negative-pressure wound therapy in latissimus dorsi flap donor site seroma prevention: A cohort study. Arch Plast Surg 44: 308-312, 2017.

17. Philip B, McCluskey P and Hinchion J: Experience using closed incision negative pressure wound therapy in sternotomy patients. J Wound Care 26: 491-495, 2017.

18. Masters JPM, Achten J, Cook J, Dritsaki M, Sansom L and Costa ML: Randomised controlled feasibility trial of standard wound management versus negative-pressure wound therapy in the treatment of adult patients having surgical incisions for hip fractures. BMJ Open 8: e020632, 2018.

19. Saku I, Kanda S, Saito T, Fukushima T and Akiyama T: Wound management with negative pressure wound therapy in postoperative infection after open reconstruction of chronic Achilles tendon rupture. Int J Surg Case Rep 37: 106-108, 2017.

20. Kaushik D, Joshi N, Kumar R, Gaba S, Sapra R and Kumar K: Negative pressure wound therapy versus gauze dressings for the treatment of contaminated traumatic wounds. J Wound Care 26: 600-606, 2017.

21. Novelli G, Daleffe F, Birra G, Canzi G, Mazzoleni F, Boni P, Maino C, Giussani C, Sozzi D and Bozzetti A: Negative pressure wound therapy in complex cranio-maxillofacial and cervical wounds. Int Wound J 15: 16-23, 2018.

22. Ludolph I, Fried FW, Kneppe K, Arkudas A, Schmitz M and Horch RE: Negative pressure wound treatment with computer-controlled irrigation/instillation decreases bacterial load in contaminated wounds and facilitates wound closure. Int Wound J 15: 978-984, 2018.

23. Bi H, Li J, Xue C and Marks M: early closure of infected laparotomy wound with negative-pressure wound therapy: Safety and efficacy in 42 consecutive cases. Am Surg 84: 938-946, 2018

24. Bazaliński D, Więch P, Barańska B and Binkowska-Bury M: Use of negative pressure wound therapy in a chronic leg wound with coexisting rheumatoid arthritis: A case study. J Int Med Res 46: 2495-2499, 2018.

25. Izadpanah K, Hansen S, Six-Merker J, Helwig P, Südkamp NP and Schmal H: Factors influencing treatment success of negative pressure wound therapy in patients with postoperative infections after osteosynthetic fracture fixation. BMC Musculoskelet Disord 18: 247, 2017. 